\title{
LE MODÈLE BELGE DE CONCERTATION BUDGÉTAIRE ET LES POUVOIRS LOCAUX
}

\author{
Jean-François Husson
}

De Boeck Supérieur | Reflets et perspectives de la vie économique

\author{
2008/4 - Tome XLVII \\ pages 33 à 48
}

\section{ISSN 0034-2971}

Article disponible en ligne à l'adresse:

http://www.cairn.info/revue-reflets-et-perspectives-de-la-vie-economique-2008-4-page-33.htm

Pour citer cet article :

Husson Jean-François, « Le modèle belge de concertation budgétaire et les pouvoirs locaux »,

Reflets et perspectives de la vie économique, 2008/4 Tome XLVII, p. 33-48. DOI : 10.3917/rpve.474.0033

Distribution électronique Cairn.info pour De Boeck Supérieur.

(c) De Boeck Supérieur. Tous droits réservés pour tous pays.

La reproduction ou représentation de cet article, notamment par photocopie, n'est autorisée que dans les limites des conditions générales d'utilisation du site ou, le cas échéant, des conditions générales de la licence souscrite par votre établissement. Toute autre reproduction ou représentation, en tout ou partie, sous quelque forme et de quelque manière que ce soit, est interdite sauf accord préalable et écrit de l'éditeur, en dehors des cas prévus par la législation en vigueur en France. II est précisé que son stockage dans une base de données est également interdit. 


\title{
Le modèle belge de concertation budgétaire et les pouvoirs locaux
}

Jean-François Husson ${ }^{1}$

\begin{abstract}
The Belgian model of budget cooperation has been originally devised to maintain Belgian economic and monetary union through a close follow-up of Regions and Communities deficits and debts. Enlarged to take into account European budgetary constraints, this model has devoted a growing attention to the local sector, as detailed in this paper. Up to now, budget and debt assignments for local authorities have been agreed by the supervisory authorities - the Regions - despite the lack of available data on a regional basis. Developments could come from greater involvement and responsibility from local authorities themselves.
\end{abstract}

Codes JEL - H72 (State and local budget and expenditures), H77 (Intergovernmental relations).

Keywords - Local finance, Fiscal federalism, Intergovernmental relations.

Dans les années 1980-1990, le modèle belge de concertation budgétaire trouve son origine dans la volonté d'éviter qu'un déficit excessif de la part d'une entité fédérée ne remette en question l'unité économique et monétaire du pays. Les principaux acteurs étaient alors le gouvernement fédéral et les exécutifs (devenus gouvernements) communautaires et régionaux. Les pouvoirs locaux n'ont été véritablement pris en compte qu'à partir du milieu des années 1990 car, comme composante du secteur des administrations publiques au sens du SEC, leur capacité de financement pouvait alléger l'effort devant être consenti par les autres sous-secteurs et la mise en bourse de Dexia fut considérée comme du pain béni pour la diminution de la dette publique brute consolidée. La présente contribution propose de parcourir rapidement les origines de cette concertation budgétaire "à la belge » ainsi que d'en décrire le fonctionnement et les développements récents en matière de pouvoirs locaux².

1. Ancien conseiller des finances, secrétaire général du Centre Interuniversitaire de Formation Permanente (CIFoP), chercheur associé au CRIS-Université de Liège, enseignant à la Haute Ecole de Namur et membre scientifique d'EUROPA.

2. Cet article est basé sur une contribution au colloque « Le pilotage macro-budgétaire des finances locales » organisé par le CIFoP à Bruxelles, le 25 juin 2008. 


\section{UNE CONCERTATION BUDGÉTAIRE ORIENTÉE VERS LA FONCTION DE STABILISATION}

Pour reprendre la traditionnelle typologie de Musgrave (1989: 3-14), les principales fonctions des pouvoirs publics sont l'allocation, la redistribution et la stabilisation, cette dernière visant les grands équilibres macroéconomiques (emploi, prix, comptes extérieurs) et une croissance raisonnable. Les outils d'une politique de stabilisation sont les instruments monétaires et les instruments budgétaires (dépenses et recettes publiques), Musgrave soulignant l'importance d'un déficit sous contrôle ; il considérait également (1989 : 455) que la politique de stabilisation devait être dans les mains de l'autorité nationale, une abondante littérature ayant par la suite souligné que la décentralisation pouvait compliquer la politique macroéconomique de stabilisation en raison d'actions non coordonnées de l'autorité nationale et des autorités infranationales ${ }^{3}$.

Mise en place dans l'optique de préserver l'union économique et monétaire belge, que des déficits trop importants de l'une ou l'autre entité fédérée auraient menacée, la concertation budgétaire en Belgique découlant de la loi du 16 janvier 1989 de financement des Communautés et des Régions s'inscrit principalement dans le cadre de la fonction de stabilisation en organisant le suivi attentif des déficits et de l'endettement des entités fédérées, celles-ci ayant reçu au fil des successives réformes de l'État des compétences croissantes et même une certaine autonomie fiscale. Les procédures mises en place visent à une stabilisation, voire une réduction, de l'endettement des entités fédérées.

La concertation budgétaire a acquis une nouvelle dimension avec le processus d'intégration européenne et le traité de Maastricht, prévoyant l'instauration de la monnaie unique. Pour pouvoir accéder à l'euro, les pays candidats devaient rencontrer cinq critères dont deux en matière de finances publiques (solde de financement et taux d'endettement). Ces objectifs, tant dans le cadre du déficit (besoin net de financement n'excédant pas $3 \%$ du PIB) que du taux d'endettement (ne dépassant pas $60 \%$ du PIB ou se réduisant à un rythme satisfaisant), portaient sur l'ensemble du secteur des administrations publiques, tel que défini en comptabilité nationale ${ }^{4}$, c'est-à-dire le pouvoir fédéral, les administrations de sécurité sociale, les entités fédérées et les pouvoirs locaux. Comme le soulignent Van de Voorde et Stienlet (1995 : 266), « en vue d'assurer l'efficacité de la procédure concernant les déficits excessifs, les gouvernements des États membres sont responsables des déficits du gouvernement général. Les États membres veillent à ce que les procédures nationales en matière budgétaire leur permettent de remplir les obligations qui leur incombent dans ce domaine ${ }^{5}$.

Le graphique 1 résume l'addition de ces deux jeux de contraintes ; pour les entités fédérées, il a été considéré à partir de 1992 que le respect des contraintes budgétaires intrabelges représentait la contribution des entités fédérées aux

3. Thornton et Mati (2008) ont toutefois relativisé cet argument sur la base d'une étude portant sur les pays de l'OCDE pour la période 1970-2001.

4. SEC 1979 puis le SEC95.

5. Protocole $n^{\circ} 5$ sur la procédure concernant les déficits excessifs, art. 3. 
contraintes budgétaires européennes, l'ajustement devant être fait par l'État fédéral - les pouvoirs locaux étant, à l'origine, considérés à l'équilibre ${ }^{6}$.

\section{Graphique 1 : L'addition des contraintes budgétaires}

\begin{tabular}{|c|c|c|}
\hline $\begin{array}{c}\text { Contraintes budgétaires } \\
\text { intrabelges }\end{array}$ & + & Contraintes budgétaires européennes \\
\hline Communautés et Régions & & $\begin{array}{c}\text { Secteur des administrations publiques : État fédéral, } \\
\text { Sécurité sociale, entités fédérées, pouvoirs locaux }\end{array}$ \\
\cline { 2 - 3 } $\begin{array}{c}\text { Trajectoires budgétaires en milliards BEF/ } \\
\text { millions } € \text {, sur base des rapports CSF }\end{array}$ & $\begin{array}{r}\text { Solde de financement et } \\
\text { taux d'endettement en \% du PIB }\end{array}$ \\
\hline
\end{tabular}

La prise en compte des contraintes européennes dans la concertation budgétaire a ainsi débouché sur une visibilité et une attention croissante accordées aux pouvoirs locaux. Le présent article vise donc à resituer la place accordée aux pouvoirs locaux dans le processus de concertation budgétaire belge, plus particulièrement depuis le passage à l'euro.

Par contre, ne seront pas abordées :

- la coordination des politiques budgétaires, qui consisterait par exemple en des politiques de soutien à la croissance coordonnées entre Régions et autorité fédérale ${ }^{7}$;

- la prise en compte du coût budgétaire du vieillissement ;

- la coordination des politiques fiscales, imposée notamment sur divers impôts régionaux par la loi spéciale de financement dans l'optique d'éviter une concurrence fiscale entre Régions.

\section{L'INSCRIPTION DES FINANCES LOCALES À L'AGENDA POLITIQUE ${ }^{8}$}

Jusqu'au début des années 1990, les finances locales n'ont apparemment été inscrites à l'agenda politique du pouvoir national, puis fédéral, qu'en un nombre de cas relativement limités. II s'agissait principalement de :

6. Comme le précise l'accord (de coopération) de 1996 : "Les parties au présent accord constatent, sur la base du rapport annuel 1996 de la section «Besoins de Financement des Pouvoirs publics », que la réduction du besoin net de financement (BNF) de l'entité Il garantit la contribution de cette entité à l'assainissement de l'ensemble des pouvoirs publics. »

7. Cela apparaît n'avoir été que ponctuellement le cas, au travers d'un nombre limité d'accords en conférence interministérielle des Finances et du Budget (ex. : tentatives de mesures concertées en matière de transmission d'entreprises, droits de successions dans les trois Régions et droits de donation au niveau fédéral, à la fin des années 1990), voire au sein du Comité de concertation (soutien à l'industrie aéronautique). Une telle approche ne serait pourtant pas dénuée d'intérêt, les pouvoirs locaux représentant environ la moitié des investissements publics en Belgique.

8. Pour Padioleau (1982), l'accès à l'agenda politique concerne « l'ensemble des problèmes perçus comme appelant un débat public, voire l'intervention des autorités politiques légitimes ». 
- supprimer une disposition fiscale locale considérée comme un frein au développement de l'activité économique (cf. l'abolition des octrois en 1860 ou, plus récemment, les décisions régionales concernant certaines taxes communales portant sur l'activité économique) ;

- trouver une marge budgétaire du côté des pouvoirs locaux lorsque l'autorité nationale était confrontée à des difficultés en la matière ; ce fut régulièrement le cas avec une adaptation insuffisante de la dotation du Fonds des communes (p. ex. à la suite des modes de répartition introduits en 1922, 1931, 1948, 1964 - voir aussi Husson, 1990 et 2004) ;

- redresser la situation lors de la perception d'un dérapage des finances locales (ex. : instauration d'une obligation d'équilibre au service ordinaire en 1982 ; groupe de travail spécifique du Comité de concertation sur la problématique des finances locales en 2001 ; dispositions concernant les plans de gestion notamment avec le plan Tonus 2 - en Région wallonne, etc.).

Cependant, à côté de ces prises en considération ponctuelles, les pouvoirs locaux étaient également concernés implicitement par le processus de concertation budgétaire « intrabelge » mis en place en 1989, en raison de leur poids dans les budgets régionaux et communautaires:

- les Régions sont compétentes pour le financement général des communes, les Fonds des communes respectifs représentant de l'ordre de 10 à $15 \%$ de leur budget - sans compter les autres subsides aux pouvoirs locaux ;

- les Communautés sont compétentes en matière d'enseignement et financent pour une large part l'enseignement officiel subventionné (c'est-à-dire relevant des communes et provinces) ; les seules subventions traitements et subventions de fonctionnement de l'enseignement fondamental officiel subventionné représentent, par exemple, près de $10 \%$ du budget de la Communauté française ${ }^{9}$.

Assez logiquement, les entités fédérées doivent donc intégrer ces transferts aux pouvoirs locaux, comme leurs autres postes de dépenses, dans leurs arbitrages budgétaires destinés à répondre aux engagements intrabelges en matière de soldes budgétaires. À ce stade, il n'y a guère de visibilité du secteur local, lequel est " noyé » dans l'ensemble des divisions organiques, programmes et allocations de base des diverses entités fédérées ${ }^{10}$.

II en va différemment dans le cadre du respect des engagements européens. Les pouvoirs locaux sont un sous-secteur du secteur des administrations publiques au sens du SEC : leurs résultats budgétaires et en termes de dette sont donc clairement identifiés et pris en considération à côté de ceux de l'autorité fédérale, de la Sécurité sociale et des entités fédérées ${ }^{11}$. À l'approche des échéances concernant

9. Budget initial 2008 ; cela représente la moitié de la division organique 51 consacrée à l'enseignement fondamental.

10. Citons pour mémoire diverses interventions fédérales en faveur des locaux, notamment dans le cadre de la politique fédérale des grandes villes.

11. À noter que, dans ce cas, les pouvoirs locaux sont considérés globalement et non par Région. Les questions méthodologiques relatives au sous-secteur des administrations locales ne seront pas développées ici. 
l'admission dans la zone euro, les pouvoirs locaux sont passés d'une situation que I'on pourrait qualifier de " pour mémoire » (prise en compte pour 0,0 \% de PIB dans le besoin de financement) à une situation où ils ont été - comme d'autres entités du secteur des administrations publiques - mobilisés, en particulier pour la réduction du taux d'endettement ${ }^{12}$. Ce sont toutefois les Régions et non les organes représentatifs de pouvoirs locaux, qui se sont engagés à faire respecter les objectifs assignés aux pouvoirs locaux par les accords budgétaires et les programmes de stabilité, comme le soulignait le Conseil supérieur des Finances.

"Contrairement aux autres pouvoirs tels que les Communautés et les Régions, les pouvoirs locaux ne sont liés, en ce qui concerne leurs soldes budgétaires, par aucun engagement découlant de la loi spéciale de financement des Communautés et des Régions ou par le programme de stabilité. Les Régions, autorité de tutelle, se sont toutefois engagées dans la convention de décembre 2000, à les inciter à réaliser les objectifs budgétaires du programme de stabilité 2001-2005. » (Conseil supérieur des Finances - Rapport 2002)

\section{LES ACTEURS DE LA CONCERTATION BUDGÉTAIRE}

Outre l'autorité fédérale et les entités fédérées, le processus de concertation budgétaire compte deux types d'acteurs importants : un comité d'experts, le Conseil supérieur des Finances, d'une part et deux instances intergouvernementales, le Comité de concertation et la Conférence Interministérielle des Finances et du Budget, d'autre part ${ }^{13}$.

\subsection{Le Conseil supérieur des Finances et la section "Besoins de Financement des Pouvoirs publics"}

La section «Besoins de Financement des Pouvoirs Publics » du Conseil supérieur des Finances (CSF) est instituée par l'article 49 («Des emprunts ») de la loi spéciale du 16 janvier 1989 de financement des Communautés et des Régions. Elle est composée de 12 membres choisis " en raison de leur compétence particulière et de leur expérience dans le domaine financier et économique »: six représentants des entités fédérées (la Région de Bruxelles-Capitale, la Région wallonne, la Communauté française, les Communauté et Région flamande) et 6 autres membres, dont le représentant du ministre des Finances et trois représentants de la Banque nationale de Belgique, le tout avec parité linguistique.

La section rend un avis (en fait un rapport) annuel sur les besoins de financement des pouvoirs publics. Elle peut, d'initiative ou à la demande du ministre

12. Pour une présentation détaillée, voir Husson (2001).

13. Les modalités de cette concertation sont donc clairement coopératives et non de concurrence, au contraire de ce qui se passe dans d'autres pays (voir Mignolet, 2005). 
des Finances, remettre un avis sur l'opportunité de limiter la capacité d'emprunt d'une entité fédérée " en fonction de la nécessité de ne pas porter atteinte à l'union économique et à l'unité monétaire, et d'éviter toute perturbation des équilibres monétaires internes et externes ainsi qu'une détérioration structurelle des besoins de financement ».

Dans ces rapports annuels, les pouvoirs locaux ont occupé une place variable. Un chapitre spécifique leur est consacré, notamment depuis 2000, et une attention particulière a été portée à l'impact du " cycle électoral " ${ }^{14}$. La section et son secrétariat ont également été amenés à répondre à des questions des instances internationales (FMI, Commission européenne) sur les finances locales depuis au moins 2000.

Enfin, au sein du CSF, une section sur les transferts fiscaux aux pouvoirs locaux avait été envisagée au début des années 2000 mais elle n'a finalement jamais été constituée. La section «Fiscalité » du CSF a été chargée de cette question et y a consacré un premier rapport en 2007, ainsi qu'un second rapport en 2008. Elle est en effet tenue d'émettre un avis annuel sur ce sujet.

\subsection{Le Comité de concertation}

L'instance centrale en matière de concertation et de coordination entre l'État fédéral et les entités fédérées est le Comité de concertation, créé par l'article 31 de la loi de réformes institutionnelles du 9 août 1980. Composé paritairement en termes linguistiques, il comprend le Premier ministre et cinq membres du gouvernement fédéral, le président et un membre du gouvernement flamand, le président du gouvernement de la Communauté française, le président du gouvernement wallon, le président du gouvernement de la Région de Bruxelles-Capitale et un de ses membres appartenant à l'autre groupe linguistique. La loi spéciale de financement lui confie certaines missions spécifiques, comme une concertation annuelle sur la politique fiscale (LSF, 1989, art. 8).

En matière de concertation budgétaire, le principal rôle du Comité de concertation est d'entériner les accords ou conventions négociés en Conférence Interministérielle des Finances et du Budget. Rappelons également qu'un groupe de travail spécifique sur les problèmes des finances communales a été constitué au sein du Comité de concertation en $2001^{15}$.

\subsection{La Conférence Interministérielle des Finances et du Budget (CIFB)}

"Le Comité de concertation peut, en vue de promouvoir la concertation et la coopération entre l'État, les Communautés et les Régions, constituer des comités

14. Le cycle électoral se traduit par une nette augmentation des investissements communaux à l'approche des échéances électorales communales.

15. Les résultats de ce groupe ne semblent pas avoir fait l'objet d'une publication. 
spécialisés dénommés "conférences interministérielles" composés de membres du gouvernement et des exécutifs des Communautés et des Régions " (Loi de réformes institutionnelles, 9 août 1980, art. 31 bis). La Conférence Interministérielle des Finances et du Budget (CIFB) n'a donc pas de pouvoir décisionnel propre, ses décisions devant être ratifiées par le Comité de concertation. La CIFB comprend les ministres fédéraux, régionaux et communautaires des Finances et du Budget (les ministres fédéraux du Budget et des Finances ; les ministres des Finances et du Budget de la Région wallonne, de la Communauté française, de l'autorité flamande et de la Communauté germanophone) ; le secrétariat est assuré par un membre du service d'études du SPF Finances. Elle constitue un instrument relativement informel, mais essentiel, de coordination, notamment lorsqu'il s'agit de préparer des accords en matière budgétaire en amont du Comité de concertation.

\section{LES OUTILS DE LA CONCERTATION BUDGÉTAIRE}

Plusieurs types d'outils peuvent être distingués : il y a ceux qui organisent la concertation budgétaire globale (entre autorité fédérale et entités fédérées, voire vers l'Europe - ce que représente le graphique 2) et des outils spécifiques aux pouvoirs locaux.

\section{Graphique 2 : Acteurs et outils de la concertation budgétaire}

\begin{tabular}{|c|c|c|c|c|c|}
\hline $\begin{array}{c}\text { Conseil } \\
\text { supérieur des } \\
\text { Finances }\end{array}$ & $\begin{array}{c}\text { Conférence } \\
\text { Interministérielle } \\
\text { des Finances et } \\
\text { du Budget }\end{array}$ & $\begin{array}{c}\text { Comité de } \\
\text { concertation }\end{array}$ & & \\
\hline $\begin{array}{c}\text { Rapport/avis annuel } \\
\text { sur les contraintes } \\
\text { intrabelges et } \\
\text { exécution du plan } \\
\text { de convergence/ } \\
\text { programme de } \\
\text { stabilité }\end{array}$ & $\begin{array}{c}\text { (Préparation des) } \\
\text { accords/conventions } \\
\text { sur les soldes } \\
\text { budgétaires }\end{array}$ & $\begin{array}{c}\text { Formalisation } \\
\text { des accords/ } \\
\text { conventions } \\
\text { sur les soldes } \\
\text { budgétaires }\end{array}$ & & $\begin{array}{c}\text { Plan de } \\
\text { convergence/ } \\
\text { pacte de } \\
\text { stabilité }\end{array}$ \\
\hline
\end{tabular}

\subsection{Les accords et conventions}

Selon l'article 92 bis, $\S 1$, de la loi spéciale de réformes institutionnelles du 8 août 1980, « L'État, les Communautés et les Régions peuvent conclure des accords de coopération qui portent notamment sur la création et la gestion conjointes de services et institutions communs, sur l'exercice conjoint de compétences propres, ou sur le développement d'initiatives en commun ". De tels accords doivent être ratifiés par des lois, décrets ou ordonnances. Pour faire bref, les accords de coopération sont le pendant intrabelge des traités internationaux. 
Le terme d'accord de coopération a été utilisé à plusieurs reprises pour les accords conclus en CIFB et officialisés en Comité de concertation, afin de souligner l'importance politique de ceux-ci. Ils n'ont toutefois jamais fait l'objet de loi, de décret ou d'ordonnance, s'agissant d'accords considérés comme techniques. Toujours est-il que, par la suite, les termes d'accord et de convention ont été préférés, pour éviter toute confusion.

Depuis leurs origines, ces accords portent sur les soldes budgétaires et d'autres questions liées telles que la dette ou la trésorerie. Certaines questions méthodologiques, mais lourdes de conséquences politiques, y ont également été abordées. Ces accords découlent généralement des rapports du CSF, dont ils ont régulièrement repris les objectifs ainsi que la méthodologie.

\subsection{Des plans de convergences aux programmes de stabilité}

Lors de la marche vers l'euro, les pays candidats devaient élaborer et présenter des plans de convergence, lesquels sont devenus par la suite - une fois admis dans l'euro - des programmes de stabilité. L'élaboration de ceux-ci repose sur une concertation de plus en plus développée pour rencontrer les objectifs européens d'abord, mais aussi pour intégrer des objectifs spécifiques - en particulier l'impact du coût du vieillissement. En termes opérationnels, le programme de stabilité précise les objectifs pour les entités ${ }^{16}$ et $\mid{ }^{17}$ et leurs composantes (dont les pouvoirs locaux). Par ce plan, le pouvoir fédéral s'engage pour l'ensemble des pouvoirs publics du pays. Depuis le plan de convergence de la Belgique adopté en $1992^{18}$, portant sur les années 1993 à 1996, la tâche d'en vérifier le respect a été confiée à la section "Besoins de financement » du CSF ${ }^{19}$.

\subsection{Les outils spécifiques aux pouvoirs locaux}

Un outil spécifique aux pouvoirs locaux concerne les normes de l'autorité supérieure et la tutelle. II s'agit là d'outils antérieurs à l'actuel débat de pilotage macrobudgétaire, mais qui contribuent à celui-ci, par l'addition de décisions relatives à des cas individuels. La suite de l'exposé se basera principalement sur la situation en Région wallonne ; des mesures différentes, mais concourant aux mêmes objectifs, ayant été prises en Flandre et en Région de Bruxelles-Capitale.

Avant de décrire ces outils, il convient de rappeler certaines spécificités des comptes et budgets des pouvoirs locaux (communes, provinces, CPAS, zones de police), qui rendent difficiles les comparaisons avec les présentations budgétaires fédérales, communautaires ou régionales ${ }^{20}$.

16. L'entité I comporte l'autorité fédérale et la Sécurité sociale.

17. L'entité Il comporte les entités fédérées et les pouvoirs locaux.

18. Plan de convergence de la Belgique, 22 juin 1992, Chambre, 482/2 (S. E., 1991-1992) ; Sénat 419-1 (S. E., 1991-1992).

19. Voir notamment Deschamps (1994: 131-132).

20. Voir également Dubois (2008). 
- Les pouvoirs locaux n'ont pas un « budget unique », mais bien un budget comportant un service ordinaire (qui permet le fonctionnement normal, quotidien, de l'administration, y compris les charges d'emprunts - capital et intérêts) et un service extraordinaire (qui a un impact sur le patrimoine communal).

- $\quad \mathrm{Si}$, depuis 1982, la loi communale ${ }^{21}$ impose l'équilibre budgétaire, l'équilibre du budget ordinaire peut être atteint par un prélèvement sur un fonds de réserve constitué des bonis antérieurs ${ }^{22}$, mais l'équilibre du budget extraordinaire peut être obtenu en recourant à l'emprunt.

- Enfin, les pouvoirs locaux ne pratiquent pas la « règle de gestion » mais la règle " de l'exercice ", c'est-à-dire que " la date du fait ou de l'acte qui est à son origine déterminera le budget qui prendra en charge la dépense quelle que soit la date de l'ordonnancement ${ }^{23}$ : on distingue ainsi l'exercice propre, qui concerne les recettes et les dépenses de l'exercice considéré, et l'exercice global, qui intègre des opérations relatives aux exercices antérieurs et des prélèvements ${ }^{24}$.

Les deux éléments les plus importants en termes de conséquence pour le pilotage macrobudgétaire vont donc être :

- l'obligation d'équilibre du service ordinaire, ce qui implique que les charges de personnel, de fonctionnement, de transfert et les charges des emprunts soient couvertes par les recettes habituelles de la commune (fiscalité, Fonds, etc.) et l'éventuel boni des années antérieures ;

- l'exercice de la tutelle à laquelle sont soumis les pouvoirs locaux, comme « pouvoirs subordonnés » : les décisions des communes en matière de budget, de comptes, de taxes, de dette, de plans de gestion, etc., sont en effet soumises à une tutelle régionale d'approbation ${ }^{25}$; ainsi, lorsqu'une commune adopte son budget, celui-ci ne peut être mis en œuvre qu'une fois approuvé par la tutelle régionale.

La tutelle n'approuvera pas un budget communal dont le service ordinaire n'est pas en équilibre ; elle tirera également la sonnette d'alarme si un budget ne respecte pas la règle dite du tiers boni (pour faire bref, si, pour atteindre l'équilibre du service ordinaire, une commune fait appel à plus d'un tiers du boni des exercices antérieurs, la tutelle y verra un risque de détérioration de la situation et pourra, le cas échéant, imposer à la commune d'élaborer un plan de gestion). En revanche, lorsque la Région wallonne a tenté de limiter les taux d'imposition d'additionnels

21. Obligation maintenue en Région de Bruxelles-Capitale et en Région wallonne (CDLD, art. L. 1312-1) ; en Région flamande, l'accent est mis sur l'équilibre du plan financier pluriannuel (décret communal, 15 juin. 2005).

22. Le solde du budget ordinaire est donc une donnée intéressante, mais il ne peut être considéré comme un « solde net à financer » (c'est-à-dire comme une mesure de l'augmentation de la dette) puisque l'amortissement des emprunts y figure en dépense.

23. Daerden et Dumazy (1992: 145-146).

24. Pour une explication détaillée, voir notamment Dexia (2008 : 6-9), Dexia (2006 : 18-21) et Crédit Communal de Belgique, Les finances des pouvoirs locaux en 1995, p. 31.

25. Nous nous référons ici à la situation de la Région wallonne et de Bruxelles-Capitale ; la situation peut être quelque peu différente en Flandre. 
communaux ${ }^{26}$ (par ex. à l'occasion d'une circulaire budgétaire), la Cour d'arbitrage (devenue la Cour constitutionnelle) a défendu le principe de l'autonomie communale.

Si les investissements des pouvoirs locaux représentent environ la moitié des investissements publics en Belgique, ceux-ci sont également en partie encadrés par les autorités régionales : en Région wallonne, lorsqu'ils sont subsidiés, ces investissements s'inscrivent dans le cadre d'un plan d'investissement triennal élaboré par chaque commune et soumis à l'approbation de la tutelle ${ }^{27}$. Enfin, les plans de gestion (qui existent sous diverses formes dans les trois Régions) permettent de piloter les finances de communes confrontées à certains problèmes avec l'aide d'interventions régionales.

Le principal élément à retenir de cette rapide description est qu'actuellement il n'y a pas de pilotage régional global des finances communales : les divers mécanismes en place témoignent d'avantage d'une approche micro (décisions relatives à des communes considérées individuellement) et non macro.

\section{LA MISE EN OEUVRE}

Après avoir présenté les acteurs et les outils de la concertation budgétaire, nous proposons de passer en revue les références aux pouvoirs locaux dans les principaux documents de référence évoqués (rapports du CSF, accords/convention, etc.). Cet examen détaillé permet de constater une très grande diversité en termes de contenu et de modalités et de voir comment se concilient engagements belgo-belges et européens. Cette recension portera essentiellement sur la période à partir de 2000, à l'exception des accords sur les soldes budgétaires, pour lesquels nous partirons de celui de 1994, ce qui nous permettra d'évoquer la « marche vers l'euro ».

\subsection{Les pouvoirs locaux dans les accords et conventions en matière de soldes budgétaires ${ }^{28}$}

Le tableau 1 récapitule les dispositions relatives aux pouvoirs locaux figurant dans les accords (" de coopération ») et conventions successifs. Ce tableau confirme l'intérêt croissant porté aux pouvoirs locaux, tant en termes de solde de financement que d'endettement - avec le cycle électoral évoqué à l'approche de chacune des échéances électorales communales $(2000,2006)$ - et le rôle des Régions comme pouvoir de tutelle. Les objectifs en matière de pouvoirs locaux ne sont toutefois pas ventilés par Région, en dépit d'une suggestion du CSF d'explorer cette piste (cf. infra).

26. Les additionnels communaux constituent une taxe communale additionnelle qui s'applique à l'impôt des personnes physiques (qui relève de l'autorité fédérale) et au précompte immobilier (qui relève des Régions) ; les communes peuvent en fixer le taux. Un décime additionnel est également ajouté à la taxe de circulation, mais les communes ne peuvent en moduler le taux.

27. En revanche, la Région flamande a d'abord introduit un système de droits de tirage en matière d'investissements, avant d'intégrer ces moyens au Fonds des communes lors de la réforme de 2002.

28. II est regrettable que l'ensemble de ces accords et conventions ne fassent pas l'objet d'une publication systématique ou d'une mise en ligne sur le site du SPF Finances. Certains d'entre eux ont été publiés en annexe de rapports du CSF, mais pas de façon exhaustive. 


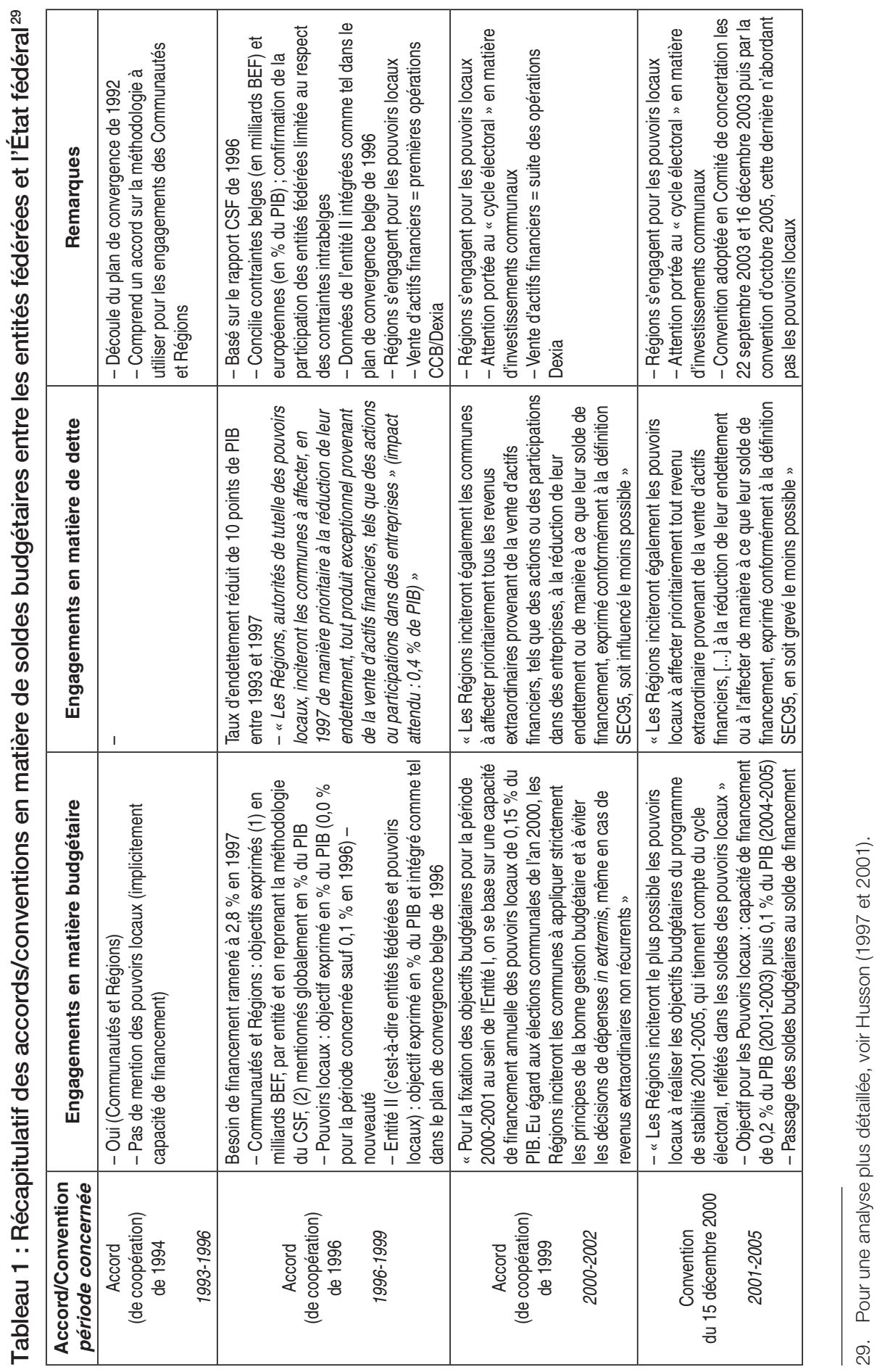




\subsection{Les pouvoirs locaux dans les rapports du CSF}

Si la part consacrée aux pouvoirs locaux dans les rapports du CSF a pu varier au fil du temps, nous souhaitons mentionner quelques extraits significatifs traitant respectivement des missions confiées aux communes et du coût du vieillissement ; de problèmes à la fois institutionnels et méthodologiques concernant l'articulation entre finances locales et régionales ; de l'évolution du solde budgétaire des pouvoirs locaux, au vu - principalement - du " cycle électoral ».

Le rapport de 2002 met les finances locales en perspective, qu'il s'agisse des tâches confiées aux pouvoirs locaux ou du coût budgétaire du vieillissement : "Le respect du résultat imparti aux pouvoirs locaux est l'un des points critiques du programme de stabilité. Divers indicateurs soulignent qu'à politique inchangée la situation financière des pouvoirs locaux connaîtra probablement une détérioration structurelle à moyen terme, qui se manifestera à partir de 2004, par une dégradation du solde courant des pouvoirs locaux. Les pouvoirs locaux devront faire face, dans un avenir proche, à d'importants défis financiers, qui influenceront leurs recettes à la baisse et leurs dépenses à la hausse. C'est pourquoi l'autorité de tutelle doit veiller à ce que l'élargissement de l'ensemble de tâches aille de paire avec une augmentation correspondante des moyens. À l'instar de ce qu'a fait le pouvoir fédéral lors de la création du Fonds de vieillissement, les communes feraient bien de constituer aussitôt que possible des réserves (constituées notamment de recettes non récurrentes) destinées au financement des pensions futures en alimentant les fonds de pension locaux, ce qu'elles n'ont fait jusqu'à présent que dans une mesure insuffisante. "

Le rapport de 2003 souligne le lien entre les pouvoirs locaux et les Régions : "Étant donné la grande interdépendance qui existe entre les administrations locales et les administrations régionales, il est souhaitable de pouvoir apprécier les évolutions budgétaires de ces entités sur une base régionale consolidée. La section a formulé une demande en ce sens à l'ICN [...]. »30 Derrière cette considération institutionnelle se profilent également des préoccupations en termes de données et de méthodologie ; dans le même registre, le rapport 2004 évoquera la révision des "statistiques afférentes à la dette Maastricht des administrations locales » afin de répondre aux demandes d'Eurostat tandis que le rapport 2007 signale que " la transmission rapide de données exhaustives et cohérentes nécessaires à l'élaboration du compte des administrations locales reste problématique, ce qui peut occasionner des révisions des comptes de ce sous-secteur. La section ne peut dès lors que réitérer sa demande d'une centralisation et d'une consolidation cohérente des données par les autorités de tutelle de manière à améliorer la qualité des comptes et à permettre une décomposition régionale de ceux-ci. »

D'autres rapports font surtout porter l'attention sur le résultat budgétaire et le cycle budgétaire. Ainsi, dans son rapport 2004, " la section recommande [...] que les différentes Régions restent vigilantes par rapport à l'évolution des dépenses des administrations locales sur lesquelles elles exercent un pouvoir de tutelle ». Le rapport de 2007 revient sur le cycle électoral : "La progression des dépenses en capital s'élève à 0,3 \% du PIB et correspond au cycle électoral

30. De telles données régionales ne sont toujours pas disponibles à ce jour. 
traditionnel où une augmentation particulière des dépenses se manifeste au cours de l'année des élections communales et au cours de l'année qui précède. Les effets du cycle électoral sont mis en lumière en calculant l'écart des dépenses effectives, exprimées en \% du PIB, par rapport à leur tendance de longue période. En tenant compte de ces écarts les dépenses en capital sont tendanciellement stables à prix constants, en \% du PIB tendanciel, à 0,8 \% de PIB. » Le rapport 2008 constate pour sa part que " contrairement à 2006, on n'identifie pas en 2007 d'incidence du cycle "politique ou électoral" des dépenses d'investissements des pouvoirs locaux par rapport à leur trend de long terme ».

\subsection{Les pouvoirs locaux dans les programmes de stabilité}

Nous nous bornerons ici au programme de stabilité 2008-2011 ${ }^{31}$, qui mentionne : "Le gouvernement veut atteindre cet objectif [de $1 \%$ de PIB d'excédent structurel en fin de législature - 2011] par un juste équilibre entre la maîtrise des dépenses et la croissance des recettes. En concertation avec les Régions et les Communautés, il fixera la contribution que chaque niveau de pouvoir, y compris les administrations locales, devra apporter à la réalisation de cet objectif commun. »

L'objectif assigné aux pouvoirs locaux en matière de solde de financement est ainsi de 0,0 \% du PIB en 2007, 0,1 \% en 2008, 0,2 \% en 2009 et 2010, 0,1 \% en 2011, année préélectorale au niveau local.

\section{PERSPECTIVES}

Aux termes de cet exposé essentiellement descriptif, la question centrale - rejoignant le constat opéré par le CSF dès 2002 - est de savoir comment articuler le financement des tâches attribuées aux pouvoirs locaux avec les contraintes budgétaires qui leur sont imposées, que ce soit dans un cadre décrétal et réglementaire régional, dans le cadre du respect par chaque Région de ses engagements à l'égard des autres entités fédérées et de l'État fédéral et du respect par les pouvoirs publics belges des engagements pris à l'égard de l'Union européenne et des partenaires de la zone euro. D'aucuns y ajouteraient également « une surveillance plus étroite [des] politiques fiscales des autorités locales $\gg 32$.

Comme le montrent les citations extraites des accords en matière de soldes budgétaires, des rapports du CSF et du plan de convergence, ce sont les Régions - autorités de tutelle - qui s'engagent au nom des pouvoirs locaux. Devant le constat de l'importance croissante accordée aux pouvoirs locaux, la question d'une plus grande implication et responsabilisation de leur part peut être posée.

Un obstacle déjà cité est d'ordre méthodologique : la difficulté d'obtenir des données exhaustives relatives aux pouvoirs locaux ainsi que les difficultés de passage des normes budgétaires locales aux normes SEC appliquées au secteur

31. Pour le dernier programme de stabilité (2008-2011), voir Bulletin de Documentation, Service d'études et de documentation, SPF Finances, $n^{\circ} 2$ (2008), 5-33.

32. Van Rompuy (2005 : 394). 
des administrations publiques dans le cadre du plan de convergence, ont été mentionnées, notamment par le CSF $^{33}$.

Cependant, l'organisation de pactes budgétaires régionaux initiés par chaque Région, en associant les Communautés (au vu de l'importance des interventions communautaires en matière d'enseignement, de culture et de petite enfance) et l'ensemble des pouvoirs locaux concernés ${ }^{34}$, et en permettant de piloter les finances locales d'une façon proactive et non plus ex-post, nous semble pouvoir constituer un outil intéressant afin de répondre à la question posée ci-dessus.

\section{CONCLUSIONS}

Le modèle belge de concertation budgétaire a été constitué pour le maintien de I'union économique et monétaire belge ; il a depuis évolué pour prendre en compte la contrainte budgétaire européenne et l'impact du coût du vieillissement.

Cette concertation repose sur des mécanismes coopératifs ${ }^{35}$ instaurés par les lois de réformes institutionnelles et la loi spéciale de financement de $1989^{36}$, reposant sur des acteurs tels que le CSF (comité d'experts), le Comité de concertation et la Conférence Interministérielle des Finances et du Budget (organes intergouvernementaux). Cette concertation ayant globalement bien fonctionné ${ }^{37}$, il apparaît souhaitable qu'elle soit préservée voire améliorée ${ }^{38}$.

$\mathrm{Si}$, à l'origine, les pouvoirs locaux ne recueillaient que peu d'attention dans ce processus, la prise en compte des contraintes budgétaires européennes - et l'instauration de la méthodologie SEC - ont donné une plus grande visibilité au secteur des administrations locales qui, de plus, présentait une capacité de financement et disposait d'actifs financiers pouvant être affectés à la réduction de l'endettement. Les pouvoirs locaux se sont alors vus assigner des objectifs en matière de solde de financement et d'endettement, les Régions - autorités de tutelle - s'engageant en leur nom.

Si les résultats attendus en matière de soldes budgétaires et d'endettement ont pu être atteints, cela provient a priori d'un ensemble de décisions individuelles concernant les pouvoirs locaux, les autorités régionales ne disposant pas actuellement de véritables outils de pilotage des finances locales.

33. Voir aussi Dubois (2008) et les contributions d'O. Dubois et d'A. Dessoy au colloque « Le pilotage macro-budgétaire des finances locales » organisé par le CIFoP à Bruxelles, le 25 juin 2008.

34. L'approche est donc différente des pactes locaux conclus en Flandre, convention bilatérale entre un pouvoir local et la Région.

35. Van Rompuy (2005 : 378, 381-384). Pour d'autres systèmes, voir p. ex. les cas de l'Allemagne et du Canada dans Chavellard et Guihéry (2005), ou celui du Danemark dans Rattsø et Tovmo (2008).

36. Nous n'aborderons pas ici la coopération imposée dans certains cas en matière fiscale (ex. :taxe de circulation), et qui a parfois entraîné des blocages (vignette autoroutière p. ex.).

37. Husson (1997), Van Rompuy (2005). Nous n'évoquerons pas ici le débat sur l'importance des moyens octroyés aux Communautés et Régions à l'occasion de la réforme de l'État de 2001 , " appauvrissant » ainsi l'État fédéral.

38. D'une manière générale, il conviendra d'apprécier, avec le recul, l'impact de gouvernements asymétriques, comme c'est actuellement le cas, rendant la concertation budgétaire plus difficile qu'au milieu des années 1990, quand les mêmes familles politiques étaient au pouvoir tant au niveau fédéral que dans les entités fédérées. 
Dans un souci de responsabilisation, se pose également la question d'une plus grande implication des pouvoirs locaux dans la mise en œuvre de ces objectifs en matière de soldes budgétaires et d'endettement. La mise sur pied de pactes budgétaires régionaux, associant les diverses autorités concernées, pourrait constituer une piste en la matière.

\section{BIBLIOGRAPHIE}

B. Bayenet, M. Feron, V. Gilbert et F. Thys-Clement, Le fédéralisme budgétaire : mode d'emploi, Éditions de l'Université Libre de Bruxelles, Bruxelles, 2000.

F. Chabellard et L. GuiherY, "Contrainte budgétaire forte ou relâchée : revue théorique et expériences fédérales au Canada et en Allemagne », in M. Mignolet (éd.), Le fédéralisme fiscal. Leçons de la théorie économique et expérience de quatre États fédéraux, De Boeck, Bruxelles, 2005, p. 337-375.

Conseil supérieur des FinAnCes, section « Besoins de financement des pouvoirs publics ", Rapport annuel, années 1992 à 2008 (téléchargeables sur http://docufin. fgov.be/intersalgfr/hrfcsf/adviezen/Adviezen.htm)

M. Daerden et W. Dumazy, Les finances publiques de la nouvelle Belgique fédérale, Labor, Bruxelles, 1992, 735 p.

R. DESCHAMPS, «La coordination des politiques budgétaires des différents niveaux de pouvoir en Belgique fédérale " in Finances publiques régionales et fédéralisme fiscal, Presses Universitaires de Namur, Namur, 1994, p. 127-137.

P. H. DerYCKE, «Les théories du fédéralisme fiscal et aujourd'hui », in Finances publiques régionales et fédéralisme fiscal, Presses Universitaires de Namur, Namur, 1994, p. 19-33.

DEXIA, Les élections communales 2006. Une approche thématique et statistique des finances locales, Dexia, Bruxelles, 2006, 63 p. (téléchargeable sur www.dexia.be)

DEXIA, Réforme comptable et nouveaux outils $d$ analyse financière pour les communes et les CPAS wallons, Dexia, Bruxelles, 2008, 60 p. (téléchargeable sur www.dexia.be)

O. DuBoIs, «Les trajectoires budgétaires : principes, évolutions récentes et implications pour les pouvoirs locaux ", Mouvement communal, $n^{\circ} 2$, Union des Villes et Communes de Wallonie, Namur, 2008, p. 80-90.

J.-F. Husson, "Les Accords de Coopération en matière d'objectifs budgétaires ", Bulletin de Documentation, $\mathrm{n}^{\circ} 2$ (mars-avr.), ministère des Finances, Bruxelles, 1997, p. 183-214.

J.-F. Husson et J. L. SteYLAeRS, « Les critères de convergence du traité de Maastricht et la consolidation de la dette publique ", Bulletin de Documentation, $n^{\circ} 4$ (juill.-août), ministère des Finances, Bruxelles, 1999, p. 3-45.

J.-F. Husson, "La contribution des pouvoirs locaux à la réalisation des objectifs du traité de Maastricht », Bulletin de Documentation, $n^{\circ} 1$ (janv.-févr.), ministère des Finances, Bruxelles, 2001, p. 15-55.

J.-F. Husson, Les procédures et la gestion budgétaire au niveau des collectivités locales, Rapport du Comité directeur sur la démocratie locale et régionale (CDLR), Conseil de l'Europe, cols. « Communes et Régions d'Europe », Strasbourg, 2002, 111 p. 
J.-F. Husson, "Les finances communales dans les années 1990 : une décennie de répit ? ", in É. de Callataÿ (dir.), La fin du déficit budgétaire. Analyse de l'évolution récente des finances publiques belges (1990-2000) - Histoire des finances publiques en Belgique, t. VI, De Boeck Université, Bruxelles, 2002, p. 169-190.

J.-F. Husson, "Vers une réforme du Fonds des communes en Région wallonne », Le Courrier Hebdomadaire, $n^{\circ}$ 1849-1850, Centre de Recherche et d'Information Socio-Politique (CRISP), Bruxelles, 2004, 74 p.

M. Mignolet (éd.), Le fédéralisme fiscal. Leçons de la théorie économique et expérience de 4 États fédéraux, De Boeck, Bruxelles, 2005, 410 p.

J. PAdioleau (1982), L'État au concret, PUF, Paris, 222 p.

J. Rattsø et P. Tovmo, "Fiscal Discipline and Asymmetric Adjustment of Revenues and Expenditures: Local Government Responses to Shocks in Denmark", Public Finance Review, $\mathrm{n}^{\circ}$ 30, 2002, p. 208-234.

J. Thornton et A. Mati, "Fiscal Institutions and the Relation between Central and SubNational Government Fiscal Balances", Public Finance Review, $\mathrm{n}^{\circ}$ 36, 2008, p. 243-254.

A. Van de Voorde et G. Stienlet, Le Budget de l'État dans la Belgique fédérale, 5e éd., CEPESS, Bruxelles, 1995, 308 p.

P. VAN RompuY, " La coordination des politiques budgétaires en Belgique : 15 ans d'expérience du Conseil supérieur des Finances ", in M. Mignolet (éd.), Le fédéralisme fiscal. Leçons de la théorie économique et expérience de 4 États fédéraux, De Boeck, Bruxelles, 2005, p. 377-395.

\section{ANNEXE 1}

\section{DONNÉES DU SECTEUR DES ADMINISTRATIONS LOCALES (S1313)}

\begin{tabular}{|c|c|c|c|c|c|c|c|}
\hline & \multicolumn{3}{|c|}{$\begin{array}{c}\text { Solde de financement } \\
\text { (+ : capacité de financement } \\
\text { - : besoin de financement) }\end{array}$} & \multicolumn{4}{|c|}{ Endettement } \\
\hline & \multicolumn{2}{|c|}{$\begin{array}{c}\text { Secteur Administrations } \\
\text { publiques (définition } \\
\text { Maastricht) }\end{array}$} & $\begin{array}{l}\text { dont sous-secteur } \\
\text { des administrations } \\
\text { locales }\end{array}$ & \multicolumn{2}{|c|}{$\begin{array}{l}\text { Secteur Administrations } \\
\text { publiques }\end{array}$} & \multicolumn{2}{|c|}{$\begin{array}{c}\text { dont sous-secteur } \\
\text { des administrations } \\
\text { locales }\end{array}$} \\
\hline & $\begin{array}{c}\text { En } \\
\text { millions } €\end{array}$ & $\begin{array}{l}\text { En } \% \\
\text { du PIB }\end{array}$ & $\begin{array}{c}\text { En } \\
\text { millions } €\end{array}$ & $\begin{array}{c}\text { En } \\
\text { millions } €\end{array}$ & $\begin{array}{l}\text { En \% } \\
\text { du PIB }\end{array}$ & $\begin{array}{c}\text { En } \\
\text { millions } €\end{array}$ & \\
\hline 2007 & $-965,8$ & $-0,3$ & 1823,9 & 280958 & 83,9 & 16861 & \\
\hline 2006 & 1093,1 & 0,3 & 1266,8 & 279467 & 87,8 & 16410 & \\
\hline 2005 & $-7749,5$ & $-2,6$ & $-10,9$ & 278392 & 92,1 & 15747 & \\
\hline 2004 & -532 & $-0,2$ & 153,6 & 273173 & 94,3 & 15677 & \\
\hline 2003 & $-170,4$ & $-0,1$ & $-737,1$ & 271215 & 98,7 & 14860 & \\
\hline 2002 & $-122,7$ & 0 & 1359,6 & 277130 & 103,5 & 14446 & \\
\hline 2001 & 1172,7 & 0,5 & 1794,9 & 275827 & 106,5 & 14179 & \\
\hline 2000 & 41 & 0 & 1345,3 & 271291 & 107,8 & 13213 & \\
\hline 1999 & $-1406,5$ & $-0,6$ & 1553,8 & 270679 & 113,6 & 12163 & \\
\hline
\end{tabular}

Source : Belgostat, consulté le 31 octobre 2008. 